\title{
Nívels de vale de cIclosporina elevados em transplantados RENAIS ANTI-HCV POSITIVOS
}

\author{
Luciano Wolffenbüttel*, Elsa Alídia Petry Gonçalves, Roberto Ceratti Manfro, luiz Felipe Santos Gonçalves \\ Trabalho realizado no Hospital de Clínicas de Porto Alegre - Programa de Pós-Graduação \\ em Ciências Médicas: Nefrologia - Universidade Federal do Rio Grande do Sul
}

RESUMO - Objetivo. Comparar os níveis de vale de CsA de transplantados renais anti-HCV+ com um grupo controle.

Métodos. Incluímos como casos todos os pacientes anti-HCV+ transplantados entre janeiro de 1992 e abril de 1996, e os anti-HCV. transplantados a seguir do caso como controles. Excluímos pacientes diabéticos, $\mathrm{Hbs} A g+$, os que recebiam fármacos com interação com a CsA e aqueles com transaminases elevadas. A sorologia para HCV foi testada pelo método ELISA de $3^{\mathrm{a}}$ geração, e as dosagens de ciclosporina através de fluorimetria polarizada com anticorpo policlonal.

Resultados. As principais variáveis demográficas não diferiram entre os grupos. 0 nível de vale médio de CsA do primeiro mês pós- transplante foi maior nos 23 pacientes anti-HCV $+(55 \mathrm{I} \pm 280 \mathrm{ng} /$ $\mathrm{ml})$ do que nos $3 \mathrm{l}$ controles $(418 \pm 228 \mathrm{ng} / \mathrm{ml}, \mathrm{p}<0,05)$. As diferenças tornaram-se aparentes ao final da primeira semana (528 \pm 275 versus $344 \pm 283 \mathrm{ng} / \mathrm{ml} ; \mathrm{p}<0,01$ ) e persistiam no momento da alta $(582 \pm 284 \mathrm{ng} / \mathrm{ml}$ versus $457 \pm 229 \mathrm{ng} / \mathrm{ml} ; p=0,08)$.

Conclusão. Os pacientes anti-HCV+ apresentam níveis de vale de CsA elevados em relação à população controle, 0 que indica a realização de estudo farmacocinético da droga neste prevalente grupo de transplantados renais.

UnIteRmos: Ciclosporina A. Hepatite C. Farmacocinética. Transplante renal.

\section{INTRODUÇÃO}

A ciclosporina (CsA), apesar da introdução de novas drogas imunossupressoras, permanece amplamente utilizada nos protocolos de imunossupressão da maioria dos centros de transplante renal. Já que o fármaco apresenta faixa terapêutica estreita e grande variabilidade farmacocinética intra e interindividual, é importante que sejam conhecidos todos os fatores com influência na sua farmacocinética, pois uma maior ou menor exposição à droga pode repercutir negativamente na sobrevida do enxerto.

Em 1989, o vírus da hepatite $\mathrm{C}$ (VHC) foi clonado e identificado como a maior causa de hepatite não $A$ - não $B$ transmitida de forma parenteral'. A prevalência da infecção na população em geral varia em diferentes países e áreas geográficas. $\mathrm{Na}$ população em hemodiálise, e consequentemente nos transplantados renais, esta prevalência é muito maior, atingindo, respectivamente, $28,8 \%^{2}$ e $39,5 \%^{3}$ dos pacientes em Porto Alegre, Brasil.

*Correspondência:

Rua Ramiro Barcelos, 2350 - CEP: 90035-007 Porto Alegre - RS
Observamos entre os transplantados renais do Hospital de Clínicas de Porto Alegre alguns casos de pacientes anti$\mathrm{HCV}+$ com marcanto elevação dos níveis de vale de CsA, apesar de não apresentarem clinicamente insuficiência hepática. Poucos dados a esse respeito são disponíveis na literatura. Por ser droga metabolizada pelo citocromo P450-3A-IV, os pacientes com franca insuficiência hepatocelular apresentam uma marcante elevação nos níveis de vale da CsA. Por outro lado, um estudo com 128 pacientes transplantados renais implicou a infecção pelo $\mathrm{HCV}$ como um fator predisponente à hepatite medicamentosa pela $\mathrm{CsA}^{4}$. Como não há dados sobre a farmacocinética da droga em pacientes transplantados renais portadores do HCV, mas sem insuficiência hepática franca, e esta é uma situação comum, resolvemos comparar o nível de vale da CsA destes pacientes com um grupo controle anti-HCV-.

\section{MÉtodos}

Foram estudados retrospectivamente todos os pacientes submetidos à transplante renal no Hospital de Clínicas de Porto Alegre entre janeiro de 1992 e abril de 1996.
Foram incluídos aqueles pacientes que receberam ciclosporina como base da imunossupressão (dose inicial de $4 \mathrm{mg} / \mathrm{kg} 2 \mathrm{x}$ ) dia, administrada na forma líquida com leite) por pelo menos 15 dias pós-operatório, e que tinham no mínimo duas dosagens sangüíneas de $\mathrm{Cs} A$. Consideramos casos os pacientes persistentemente anti-HCV + há pelo menos seis meses antes do transplante, e como controle o próximo paciente transplantado anti-HCV-. Os pacientes foram testados para HCV mais de uma vez enquanto em hemodiálise (mensalmente os anti-HCV- e semestralmente os anti$\mathrm{HCV}+$ ). A dose de $\mathrm{CsA}$ era rotineiramente diminuída para $6 \mathrm{mg} / \mathrm{kg}$ no caso de disfunção primária do enxerto ou no $15^{\circ}$ dia pós-transplante, e ajustadas de acordo com episódios de disfunção do enxerto ou dos níveis sangüíneos obtidos imediatamente antes da próxima dose, as quais eram efetuados duas vezes por semana. Foram excluídos os pacientes $\mathrm{HbsAg}+$, com alteração das transaminases, bilirrubinas, albumina ou tempo de pró-trombina, bem como os diabéticos, com qualquer possível causa de máabsorção (diarréia, intestino curto), com passado de abuso de álcool ou que estivessem usando alguma droga com conhecida influência na farmacocinética da CsA. 
Tabela I - Dados demográficos e laboratoriais. Comparação entre os grupos anti-HCV positivo e controle

\begin{tabular}{|c|c|c|c|}
\hline $\begin{array}{l}\text { Variável } \\
\text { (unidade) }\end{array}$ & $\begin{array}{c}\text { Anti-HCV }+(n=23) \\
\text { média } \pm \text { DP }\end{array}$ & $\begin{array}{c}\text { Anti-HCV }-(n=3 I) \\
\text { média } \pm D P\end{array}$ & $p$ \\
\hline Gênero(M/F) & $14 / 9$ & $15 / 16$ & $0,32 *$ \\
\hline $\operatorname{Raça}(B / N)$ & $19 / 4$ & $28 / 3$ & $0,50 *$ \\
\hline Doador(vivo/cadáver) & $|2 / 1|$ & $14 / 17$ & 1.00 \\
\hline Idade(anos) & $39,3 \pm 10,8$ & $37,4 \pm \pm 11,8$ & $0,48 * *$ \\
\hline Peso $(\mathrm{Kg})^{+}$ & $66,5 \pm 13,9$ & $63,8 \pm 14,2$ & $0,30 *$ \\
\hline TGP(UI) & $16 \pm 14$ & $16,3 \pm 17,2$ & $0,70 * *$ \\
\hline TGO(UI) & $11,5 \pm 5,6$ & $13,9 \pm 12,7$ & $0,43 * *$ \\
\hline Bil. total (mg/dl) & $0,63 \pm 0,19$ & $0,66 \pm 0,22$ & 0,66 ** \\
\hline Albumina (mg/dl) & $3,4 \pm 0,6$ & $3,4 \pm 0,5$ & $0,63 * *$ \\
\hline Colesteroltotal $(\mathrm{mg} / \mathrm{dl})^{+}$ & $162,3 \pm 22$ & $142,8 \pm 43,7$ & $0,33 * *$ \\
\hline Creatinina $(\mathrm{mg} / \mathrm{dl})^{++}$ & $2,3 \pm 1,6$ & $2,6 \pm 2,3$ & $0,38 * *$ \\
\hline Hematócrito $(\%)^{++}$ & $32 \pm 5,5$ & $31,3 \pm 4$ & $0,76 * *$ \\
\hline Hemoglobina $(g / d \mid)^{++}$ & $|0| \pm 3,2$, & $10,1 \pm 1,9$ & $0,97 * *$ \\
\hline
\end{tabular}

$\mathrm{M} / \mathrm{F}=$ Masculino/ feminino; $\mathrm{B} / \mathrm{N}=$ branco/ negro; $\mathrm{UI}=$ unidades internacionais; ${ }^{+}=$níveis na avaliação pré-transplante;

\begin{tabular}{|c|c|c|c|}
\hline \multirow[t]{2}{*}{ Semana (n) } & \multicolumn{2}{|c|}{ Nível de vale (ng/dl) } & \multirow[b]{2}{*}{$\mathrm{p}^{*}$} \\
\hline & $\begin{array}{c}\text { Anti- } \mathrm{HCV}+(n+23) \\
\text { média } \pm \mathrm{DP} \\
\end{array}$ & $\begin{array}{c}\text { Anti- } \mathrm{HCV}-(n=3 \mathrm{I}) \\
\text { média } \pm \mathrm{DP}\end{array}$ & \\
\hline I (84) & $528 \pm 275 * *$ & $344 \pm 283$ & $<0,01$ \\
\hline $2(96)$ & $499 \pm 243$ & $424 \pm 254$ & $=0,25$ \\
\hline $3(48)$ & $701 \pm 430$ & $500 \pm 275$ & $=0,06$ \\
\hline $4(22)$ & $781 \pm 357^{*}$ & $412 \pm 177$ & $<0,05$ \\
\hline Naalta(54) & $582 \pm 284$ & $457 \pm 229$ & $=0,08$ \\
\hline Médiamensal*** (250) & $551 \pm 280^{*}$ & $418 \pm 228$ & $<0,05$ \\
\hline
\end{tabular}

**média de todas as dosagens do primeiro mês; $(\mathrm{n})$ = número de dosagens empregadas no cálculo da média.

O teste empregado para detecção de anticorpos anti-HCV foi o ELISA de $2^{\mathrm{a}}$ ou $3^{\mathrm{a}}$ geração (Hepanostica UBI® HCV, United Biomedical Inc., Beijing). As dosagens de CsA eram feitas pelo analizador TDX®, queemprega o método de fluorimetria polarizada com anticorpos policlonais (Abbot Laboratories, Illinois, faixa terapêutica de $200-800 \mathrm{ng} / \mathrm{ml}$ ).

Para comparar variáveis contínuas foi utilizado o teste $t$ ou Mann-Whitney, e o Teste Exato de Fisher ou qui-quadrado para as variáveis categóricas. Consideramos como nível de significância um $\mathrm{p}<0,05$ para testes bicaudais. Todos os cálculos foram efetuados pelo programa Epi info.

\section{Resultados}

Após aplicarmos os critérios de exclusão para os 93 pacientes incluídos no estudo, res- taram para análise 23 pacientes anti-HCV +e 3 I controles. Não houve diferença significativa em nenhuma das características demográficas e laboratoriais estudadas (Tabela I).

As medicações concomitantes (sulfametoxazol +trimetropim, prednisona, azatioprina)e suas doses também foram semelhantes. O número de episódios de rejeiçãa aguda e disfunção primária do enxerto foram similares (dados não apresentados). Adose de CsA no momento da alta foi $6.3 \pm 1.3 \mathrm{mg} / \mathrm{kg}$ e $6.4 \pm 1.0 \mathrm{mg} / \mathrm{kg}$, respectivamente, para os pacientes anti-HCV positivos enegativos ( $p=0.48$, Mann-Whitney).

Os níveis de vale de CsA ao final de cada uma das quatro primeiras semanas pós-transplante, no momento da alta hospitalar, bem como a média do primeiro mês, estão demonstrados na Tabela 2. Os pacientes anti-HCV + apresentaram níveis superiores aos controles ao final da primeira semana $(528 \pm 275 \mathrm{ng} / \mathrm{ml}$ e $344 \pm$ $283 \mathrm{ng} / \mathrm{ml}$, respectivamente, $\mathrm{p}<0,0 \mathrm{I})$ e da quarta semana $(78 \mathrm{I} \pm 357 \mathrm{ng} / \mathrm{ml}$ e $4 \mathrm{I} 2 \pm$ $177 \mathrm{ng} / \mathrm{ml}, \mathrm{p}<0,05)$. A ciclosporinemia média do primeiro mês também foi maior nos casos $(55 \mathrm{I} \pm 280 \mathrm{ng} / \mathrm{ml})$ do que nos controles (4I8 $228 \mathrm{ng} / \mathrm{ml}, \mathrm{p}<0,05)$.

\section{Discussão}

No presente estudo foram demonstrados níveis de vale de CsA elevados em pacientes transplantados portadores crônicos de anticorpos anti-HCV, em relação a uma população controle comparável. Pacientes com condições que sabidamente influenciam a absorção e o metabolismo da CsA [5] (tais como diabetes, mal-absorção), hepatopatia clinicamente manifesta, ou portadores de hepatite $B$, foram excluídos do estudo. Além disso, condições como a idade, sexo, raça e massa corporal não diferiram entre os grupos. Os pacientes estudados são a própria população de interesse, transplantados, portadores de anti-HCV cronicamente, e usando doses usuais de CsA, conferindo validade externa (aplicabilidade) aos resultados.

A avaliação diagnóstica da infecção pelo vírus da hepatite $C$ é tema complexo. $O$ teste sorológico empregado (ELISA de segunda geração) é altamente específico e sensível para a detecção da presença de anticorpos anti-HCV, sendo desprezível a taxa de falso-positivo e falso-negativo do teste $^{6}$. No entanto, o status sorológico não confirma nem afasta infecção atual: apenas $70 \%$ a $90 \%$ dos pacientes com um teste anti-HCV reagente possuem viremia $(\mathrm{PCR}+)$ naquele momento, o que pode corresponder às seguintes situações: viremia intermitente, reservatório viral intracelular (notadamente nos hepatócitos e células mononucleares ${ }^{7}$ ) ou cicatriz sorológica (condição muito improvável, pois os anticorpos não são protetores). Em um estudo com transplantados renais, somente $70 \%$ dos pacientes com PCR+ tinham ELISA2 positivo ${ }^{6}$, o que pode deverse à imunossupressão. Portanto, a interpretação de um anti-HCV reagente aponta, na maioria dos casos, para infecção atual, com ou sem viremia. Já um teste não reagente, em transplantados renais, 
corresponde à ausência de infecção em $70 \%$ dos casos. Testagem repetida, pesquisa do RNA viral e correlação com dados clínicos aumentam o valor preditivo negativo do exame. Portanto, nossos casos possuem alta probabilidade de serem portadores atuais do HCV, e nossos controles de serem livres da infecção, já que todos os pacientes foram testados mais de uma vez e tinham persistentemente testes positivos ou negativos.

Tomados em conjunto, nossos resultados apontam para o estado de portador do vírus da hepatite $\mathrm{C}$ como a causa mais provável para os níveis de CsA elevados. O mecanismo pelo qual esse efeito foi produzido permanece obscuro. Tanto uma maior biodisponibilidade oral da CsA quanto uma menor taxa de eliminação do fármaco pelo citocromo P450-3A o produziriam. Adicionalmente, apenas os metabólitos da CsA podem estar elevados, já que o imunoensaio usado para dosagem da CsA valeu-se de anticorpos policlonais.

A biodisponibilidade oral da CsA depende da sua taxa de absorção e do metabolismo hepato-intestinal de primeira passagem. A absorção da CsA, além dos fatores já citados (diabetes, má absorção, presença de sais biliares, administração com gordura), depende da atividade da glicoproteína $P$ no epitélio enteral ${ }^{8}$. $O$ metabolismo de primeira passagem depende da atividade do citocromo P4503 A na parede intestinal e no fígado, a qual, por sua vez, varia na dependência de fatores genéticos e ambientais. Indivíduos do sexo feminino, por exemplo, apresentam uma maior atividade intestinal do citocromo P450-3A do que os homens?. Vários vírus interferem com os processos celulares de repressão e expressão gênica. $A$ presença do $\mathrm{HCV}$, levando à diminuição da expressão gênica do citocromo, poderia acarretar um aumento na biodisponibilidade da CsA.

Finalmente, a presença de disfunção hepática subclínica, a qual aumentaria a meia-vida de eliminação da droga, não pode ser descartada. No entanto, os pacientes não apresentavam indícios de dano (transaminases normais) nem de insuficiência hepatocelular (bilirrubinas, albumina e TP normais). Uma inibição viral específica dos processos de metabolização da CsA, no entanto, seria possível, e apenas estudos destinados a avaliar especificamente a atividade do citocromo P4503A poderiam esclarecer esta questão.

Recentemente, um estudo observacional com 18 pacientes transplantados renais previamente anti-HCV- que tornaram-se anti-HCV $+{ }^{10}$ demonstrou que 0 nível de vale da CsA elevou-se após a mudança do status sorológico, resultados que vêm ao encontro dos nossos. A importância destes achados repousa na possibilidade do transplantado anti-HCV+ estar submetido a uma maior exposição à droga, o que poderia ter algum impacto clínico em termos de toxicidade ou imunossupressão, como em outras subpopulações de transplantados $"$. Em recente análise do United States Renal Data System, incluindo 73.707 pacientes $^{12}$, a sobrevida do enxerto tendeu a diminuir a longo prazo nos pacientes anti-HCV+.

\section{Conclusão}

Concluímos que os níveis de vale da CsA no primeiro mês pós-transplante renal, medidos por anticorpos policlonais, estão elevados em pacientes anti-HCV+ em relação à população controle. Nossos resultados sugerem a realização de estudos farmacocinéticos controlados em pacientes anti-HCV + , a fim de confirmar estes achados, definir qual a alteração farmacocinética específica, e também de postular mecanismos explanatórios para esse achado.

\section{SUMMARY}

\section{Elevated Cyclosporine A trough LEVELS IN HCV POSITIVE KIDNEY TRANSPLANT RECIPIENTS.}

OBJECTIVE. Compare the CsA trough levels of $\mathrm{HCV}+$ kidney transplant recipients to a controlgroup

Methods. All anti-HCV positive patients that received a renal allograft between January 1992 and April 1996 were initially included as cases. Patients with diabetes mellitus, $\mathrm{HBsAg+}$, who were taking medication that could modify CsA pharmacokinetics and those with elevated aminotransferases were excluded. For each anti-HCV positive index case the following transplanted anti-HCV negative patient was included as a control. Third generation ELISA was used for determination of the anti-HCV status and $C s A$ dosages were performed by polarized fluorometry with polyclonal antibodies.

RESULTS. No differences in the demographic variables were found. The average CsA through levels in the first month were higher $(55 \mathrm{l} \pm 280 \mathrm{ng} / \mathrm{ml})$ in the 23 cases as compared to the 31 controls ( 418 $\pm 228 \mathrm{ng} / \mathrm{ml} ; p<0.05)$. The differences became apparent at the end of the first week $(528 \pm 275$ versus $344 \pm 283 \mathrm{ng} / \mathrm{ml}$; $p<0.01)$ and persisted at discharge (582 \pm 284 versus $457 \pm 229 ; p=0,08$ ).

CONCLUSION. We concluded that anti$\mathrm{HCV}$ positive patients have higher blood levels of CsA for a particular dosage, than anti-HCV negative controls. Prospective studies with a more appropriate pharmacokinetic approach are needed to confirm the present findings. [Rev Assoc Med Bras 2003; 49(2): |4I-4]

KEY wORDS: CyclosporineA. Hepatitis C. Pharmacokinetics. Kidney tranplant.

\section{REFERÊNCIAS}

I. Choo Q, Kuo G, Weiner AJ, Overby LR, Bradley DW, Houghton M. Isolation of a cDNA clone derived from a blood-borne non$A$, non- $B$, viral hepatitis genomen. Science 1989; 244:358-62.

2. Karohl C, Manfro RC, Gonçalves LF. Prevalência de anticorpos anti-vírus da hepatite $C$ em pacientes em hemodiálise crônica de Porto Alegre. J Bras Nefrol 1995; 17:40-6.

3. Manfro RC, Karohl C, Gonçalves LF, Senger MB, Thome FS, Prompt CA.. Liver function tests in hepatitis $C$ virus infected kidney transplant recipients. Transplant Proc 1995; 27:182|-2.

4. Horina JH, Wirnsberger GH, Kenner L, Holzer H, Krejs GJ. Increased susceptibility for CsA-induced hepatotoxicity in kidney graft recipients with chronic viral hepatitis $C$. Transplantation 1993; 56:1091-4.

5. Kahan BD, Kramer WG, Wideman C, Flechner SM, Lorber MI, Van Buren CT. Demographic factors affecting the pharmacokinetics of cyclosporine estimated by radioimmunoassay. Transplantation 1986; 4I:459-64.

6. Pereira BJG, Levey AS. Hepatitis C virus infection in dialysis and renal transplantation. Kidney Int 1997; 5 I:98I-99. 
7. Oesterreicher C, Hammer J, Koch U, Hsiao $\mathrm{HL}$, Turgeon DK, Brown MBet al. HBV and $\mathrm{HCV}$ genome in peripheral blood mononuclear cells in patients undergoing hemodialysis. Kidney Int 1995; 48: 1967-7I.

8. Lown KS, Mayo RR, Leichtman AB, Bader A, Kliem V, Repp $\mathrm{H}$ et al. Role of intestinal Pglycoprotein $(\mathrm{mdrl})$ in interpatient variation in the oral bioavailability of cyclosporine. Clin Pharmacol Ther 1997; 62:248-60.

9. Christians U, Bleck JS, Lampen A, et al. Are cytochrome P450 3A enzymes in the small intestine responsible for different cyclosporine metabolite patterns is stable male and female renal allograft recipients after $\mathrm{Co}$-administration of diltiazen? Transplant Proc 1996; 28:2 159-6I.

10. Tuncer M, Sülemanyar G, Ersoy FF, Yakupoglu G. Effects of hepatitis $C$ virus infection on cyclosporine trough levels in renal transplant patients. Transplant Proc 2000; 32:569-7I.

II. First MR, Schroeder TJ, Monaco AP, Simpson MA, Curtis JJ, Armenti VT. Cyclosporine bioavailability: dosing implications and impact on clinical outcomes in select transplantation subpopulations. Clin Transplant 1996; 10:55-9. 12. Ulf Meier-Kriesche H, Ojo AO, Hanson JA, Kaplan B: Hepatitis $C$ antibody status and outcomes in renal transplant recipients. Transplantation 200 I; 72:24I-4.

Artigo recebido: 10/05/2002 Aceito para publicação: II/09/2002
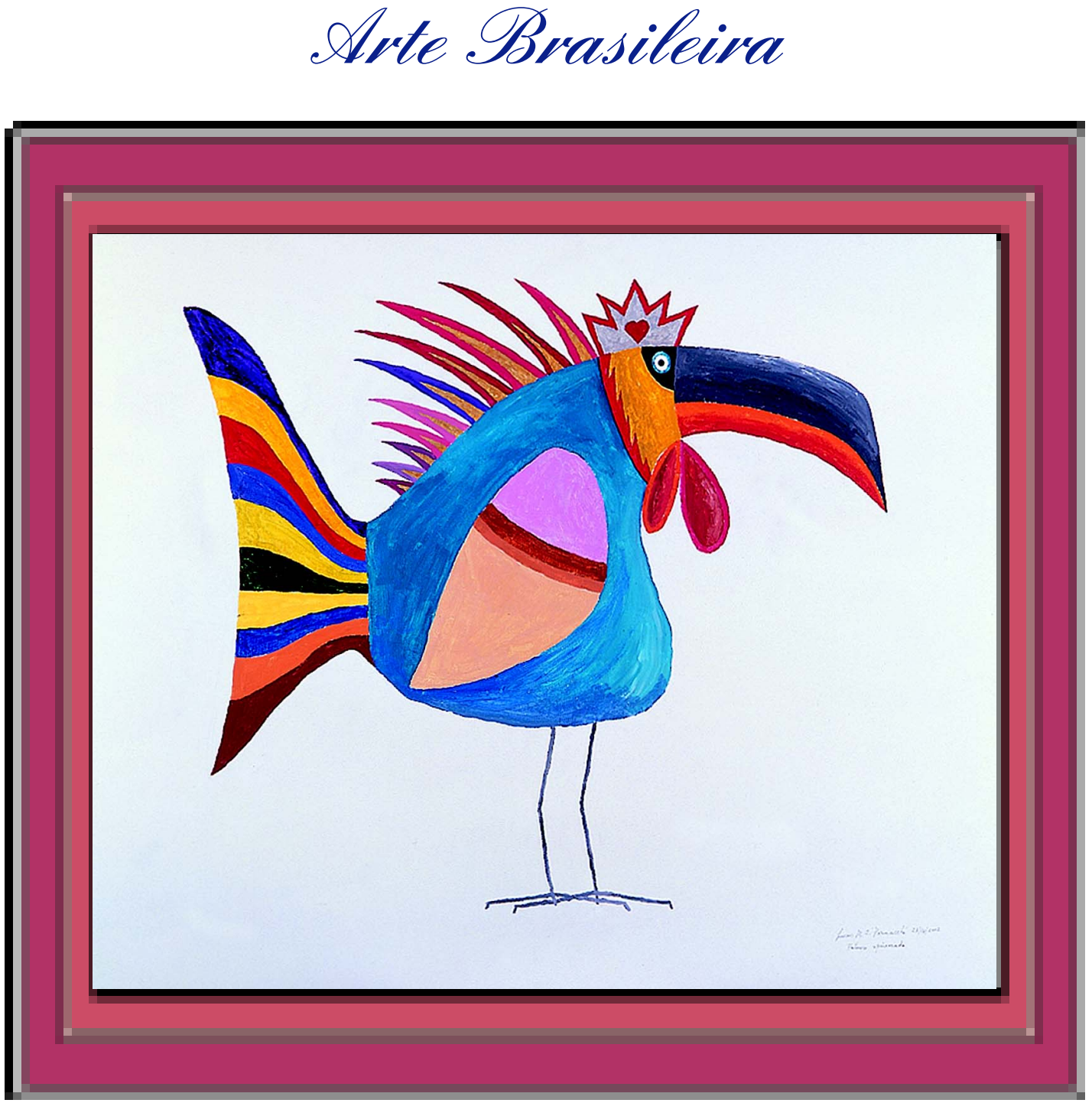

Lucas Pennacchi - "Pássaro Apaixonado" - Galeria Jacques Ardies - Tel.: (II) 3884-2916 